\title{
Electron spin resonance spectral study of PVC and XLPE insulation materials and their life time analysis
}

\author{
M.A. Morsy ${ }^{a}$,, M.H. Shwehdi ${ }^{b}$ \\ ${ }^{a}$ Chemistry Department, King Fahd University of Petroleum \& Minerals, Dhahran, Saudi Arabia \\ ${ }^{\mathrm{b}}$ Electrical Engineering Department, King Fahd University of Petroleum \& Minerals, Dhahran, Saudi Arabia
}

Received 5 January 2005; received in revised form 7 June 2005; accepted 7 June 2005

\begin{abstract}
Electron spin resonance (ESR) study is carried out to characterize thermal endurance of insulating materials used in power cable industry. The presented work provides ESR investigation and evaluation of widely used cable insulation materials, namely polyvinyl chloride (PVC) and cross-linked polyethylene (XLPE). The results confirm the fact that PVC is rapidly degrades than XLPE. The study also indicates that colorants and cable's manufacturing processes enhance the thermal resistance of the PVC. It also verifies the powerfulness and the importance of the ESR-testing of insulation materials compared to other tests assumed by International Electrotechnical Commission (IEC) Standard 216procedure, e.g. weight loss (WL), electric strength (ES) or tensile strength (TS). The estimated thermal endurance parameters by ESR-method show that the other standard methods overestimate these parameters and produce less accurate thermal life time curves of cable insulation materials.
\end{abstract}

(C) 2005 Elsevier B.V. All rights reserved.

Keywords: ESR; PVC; XLPE; Life time; Weight lose

\section{Introduction}

Polyethylene (PE) and polyvinyl chloride (PVC) are the principle materials used in the wire and cable industry. They provide insulation and jacketing for more than 30,000 different types of wire and cable products [1]. The easy processing and good insulation performance of PVC make it the polymer of choice in power cable construction. However, the recent technological improvement in insulation manufacturing trends toward using cross-linked polyethylene (XLPE) and other polymers that are more resistant to degradation from treeing than PVC in the high voltage cable construction [2-5].

During insulation manufacturing, plastics are exposed to heat that lead to degradation. The final product depends on the type of plastic, the additives used and the processing temperature. Although degradation cannot be eliminated completely, it must be held to a minimum to keep the high

\footnotetext{
* Corresponding author. Tel.: +966 3860 4761; fax: +966 38604277

E-mail address: mamorsy@kfupm.edu.sa (M.A. Morsy).
}

quality of the final products. However, the low thermal stability of PVC has been an area of intensive research, the relationship between microstructure and degradation behavior is still not fully understood.

There are many conventional and analytical testing methods available for thermal endurance characterization of insulating materials [6]. The main advantage of the analytical tests to that of the conventional methods are their abilities to reduce test times without losing accuracy and practical significance of the results. Thermo-gravimetric analysis [7-9] and relaxation measurements [10] are two well-known analytical methods. Electron spin resonance (ESR) method, also called electron paramagnetic resonance (EPR), is another analytical method that has special potential in material degradation analysis. It is well-known that ESR spectroscopy is the only technique established to detect radicals from different sources [11-13]. Also, it has been widely used in polymers degradation analysis $[14,15]$. However, it has been never used for thermal endurance characterization of manufactured and raw insulating materials used by cable industries yet. 
The present investigation will demonstrate direct testing and evaluation of thermal aging on widely used insulation materials (PVC and XLPE). The determination of thermal endurance characteristics of raw and manufactured samples from major national cable factories using ESR spectrophotometer will be conducted. The study and analysis will be carried out to simulate thermal stresses, and hence evaluate the life time property of the cable insulation materials. The results can be extended from application of life tests to more improvement and higher quality of cable's insulation materials and its manufacturing processes.

\section{Experimental}

\subsection{Materials and sample's preparation}

Raw and manufactured plastics were collected from national cable factories. The raw materials were collected from cable manufacturer while the processed materials of these plastics were taken from newly produced cable samples. The experimental tests were performed on the samples as received. The tested samples were made of tiny pieces, which were placed into glass tubes of $25 \mathrm{~mm}$ length and $2 \mathrm{~mm}$ inner diameter (i.d.) of a mass of about $0.1 \mathrm{~g}$. The tubes were tagged as given in Table 1, where the first character, R or M, stands for raw or manufactured samples, respectively, the second and the third characters were chosen to symbolize plastic's type, while the last character is for coloring condition; $\mathrm{N}$ for non-color and $\mathrm{R}$ for red-colored insulation materials.

\subsection{Thermal aging unit}

A well-design device to control thermal conditions was used to age plastic samples at 130,170 and $220^{\circ} \mathrm{C}$. The device consists of a heater tape, sample holder inside a well-isolated container that is equipped with a pre-programmable heat controller. Cole-Parmer Digi-Sense temperature controller of $0.1{ }^{\circ} \mathrm{C}$ resolution supplied power to the heater and controls its temperature as programmed using temperature sensors, $\mathrm{K}$ type thermocouple and personal computer software. External thermometer was placed inside the sample holder to show the actual temperature of the heating environment of the aging process. This was used as reference to confirm the thermal stability of the unit as function of time.

Table 1

Tagging of insulating materials used in the experiment

\begin{tabular}{lll}
\hline Sample no. & Insulating material type & Tag \\
\hline 1 & Raw ${ }^{\text {a }}$ PVC non-color & RPVN \\
2 & Raw PVC red-color & RPVR \\
3 & Manufactured ${ }^{\text {bVC red-color }}$ & MPVR \\
4 & Raw XLPE non-color & RXLN \\
5 & Manufactured XLPE non-color & MXLN \\
\hline
\end{tabular}

\footnotetext{
a Raw materials used in cable industry.

b Manufactured power cables insulation.
}

\subsection{ESR spectroscopy}

Varian E-line series spectrometer was used to detect the electron spin resonance spectra of the plastic samples. All the ESR-spectral properties of these samples were performed at room temperature using cylindrical cavity along with $9 \mathrm{GHz}$ Microwave Bridge. The working parameters of the spectrometer are as follows: time constant $=655 \mathrm{~ms}$, modulated frequency $=25 \mathrm{kHz}$, modulation amplifier $=1 \mathrm{mT}$, microwave frequency $=9.34 \mathrm{GHz}$, signal gain $=10^{4}$, sweep time $=120 \mathrm{~s}$ and sample temperature $=22^{\circ} \mathrm{C}$. These instrumental factors were carefully selected to maximize signal intensity.

\section{Results and discussion}

\subsection{ESR results}

Representative samples from the collected ESR-spectra of PVC and XLPE insulation materials during their aging process are illustrated in Fig. 1. The major difference between the non-aged and aged spectral properties is the appearance of a singlet peak in the middle region in the graphs of the deteriorated samples. Similar ESR-pattern was reported during thermal degradation [16-18], photo degradation [19,20] or chemical dehydrochlorination [21] of PVC compound. It was also observed in the $\gamma$-irradiated polyethylene compound
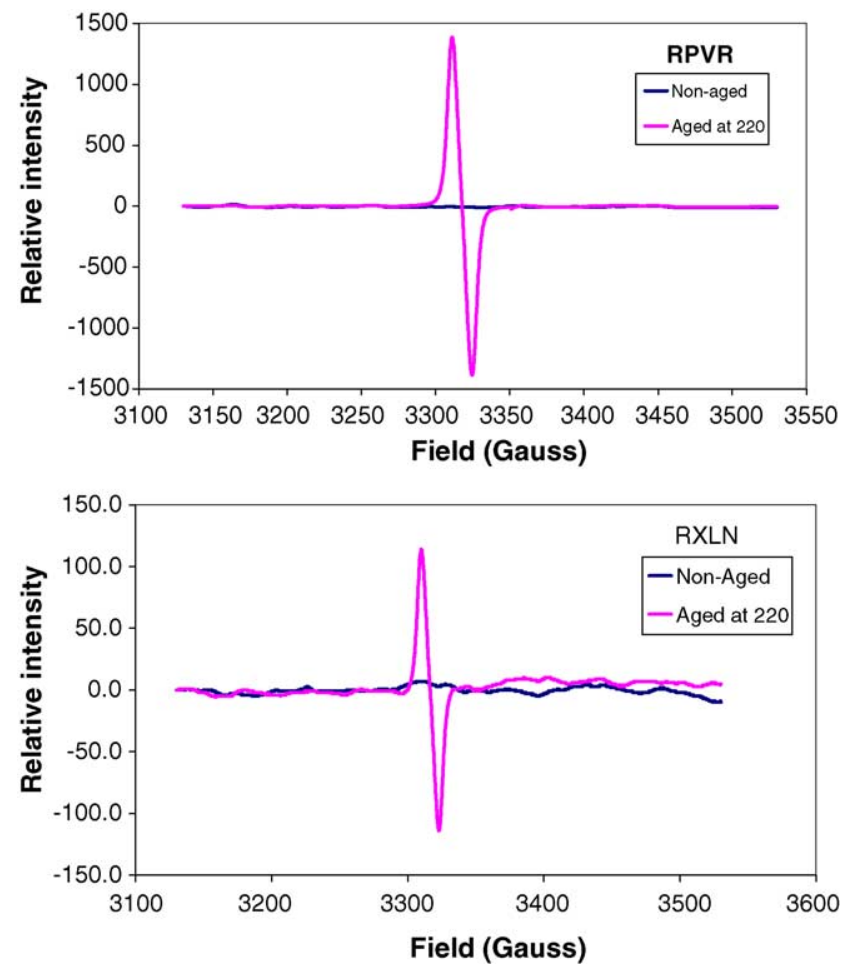

Fig. 1. Samples of ESR spectrographs of raw PVC red-colored (RPVR) and of raw XLPE non-colored (RXLN) as received (non-aged) and after aging at $220^{\circ} \mathrm{C}$ for about $23.5 \mathrm{~h}$. (For interpretation of the references to color in this figure legend, the reader is referred to the web version of the article.) 


$$
-\mathrm{CH}_{2}-\dot{\mathrm{C}} \mathrm{H}-(\mathrm{CH}=\mathrm{CH})_{\mathrm{n}}-\mathrm{CH}_{2}-
$$

Scheme 1. Proposed molecular radical of degraded plastic samples.

sample by doses of several thousands megarads [22-25]. In accordance, the appearance of the presented ESR-pattern in Fig. 1 of the degraded plastics can be attributed to the formation of conjugated polyene macroradical systems (Scheme 1) as an essential thermal degradation product.

This chemical change confirms physical changes, in general plastic degradation, which favors treeing inception and growth up to breakdown when voltage is applied [26,27]. Fig. 1 also shows that the ESR-peak intensities, which are correlated with the amount of the radical contents of the studied sample, of the aged PVC is almost 10 times the observed one of the aged XLPE sample. This high intensity of the aged PVC samples compared to XLPE is attributed to the ease of PVC degradation and is correlated very well with known stability of XLPE insulation materials toward thermal degradation relative to $\mathrm{PVC}$ materials.

Since the purpose of this experiment is to achieve thorough thermal endurance characterization of the insulation, the thermal degradation of the studied samples was diagnosed using ESR-measurements and weight loss (IEC 216 Standard method) at 130,170 and $220^{\circ} \mathrm{C}$ [28] as a function of time. In the case of ESR-measurements, series of spectral measurements were performed and then their peaks' height-to-through were plotted as a function of time of aging (Fig. 2) to pinpoint the starting of the degradation. The time of the onset points (point at which ESR-peak starts to appear) was selected as the end point or the failure time (time-to-end-point or life). Close examination of the reported results in Fig. 2 can be used to estimate the effect of cable processing and coloring additives to the insulation materials. Generally, non-linear thermal behavior of the studied plastic samples is observed, except: (1) onset of the degradation process and (2) intensity of the ESR-peak within the reported aging period. Although the peak-to-height of the produced radicals has to reflect linear relation with their amounts, the expected intermolecular interaction of these radicals to produce new materials can explain the observed non-linearity.

The detected earliest onset of degradation of raw PVC (Fig. 2) compared to the other types of plastic can be linked to the nature of additives, colorant or stabilizer and their thermal stability. Firstly, this confirms the observed high intensity of PVC-ESR-signal compared to XLPE (Fig. 1) that was attributed to the plastic stability toward thermal degradation. Secondly, the early onset of the PVC noncolored compared to PVC colored provides evidence on the selection criteria of pigments used in cable industry that is to enhance polymer stability. Moreover, a close analysis of the peak-to-through results in Fig. 2 around $800 \mathrm{~h}$ of aging period indicates that raw PVC contains about 2.5 more radical than the manufactured samples, while around and after $1500 \mathrm{~h}$
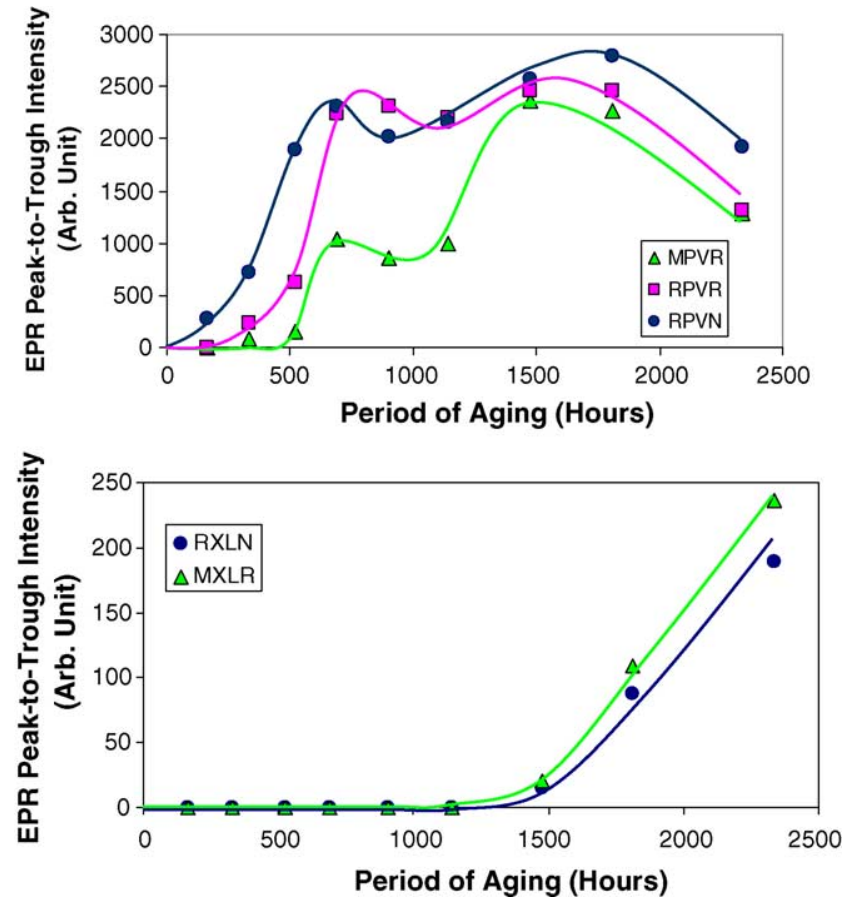

Fig. 2. Accumulation of polyene radicals per sample (raw PVC non-colored, RPVN; raw PVC red-colored, RPVR; manufactured PVC red-colored, MPVR; raw XPLE non-colored, RXLN and manufactured XLPE noncolored, MXLN) during aging at $130^{\circ} \mathrm{C}$. (For interpretation of the references to color in this figure legend, the reader is referred to the web version of the article.)

of aging period, similar heights are obtained by all PVC samples. The enhanced degradation of non-manufactured PVC samples may be attributed to the production of the corrosive $\mathrm{HCl}$-molecular system as by-product but with much large quantity than those produced from the manufactured system. On the other hand, the manufactured XLPE samples introduce more radicals than the non-manufactured one. This is correlated with absence of the $\mathrm{HCl}$ and it is also in good agreement with the known properties of the manufactured XLPE, which lose its basic properties during its manufacture processing, and hence cannot be recycled.

\subsection{Life time analysis}

In accordance with IEC Standards 216, insulating materials are classified on the basis of their "temperature index" (TI) [28] temperature corresponds to a time to reach the end point, failure criterion, of $20,000 \mathrm{~h}$. Another quantity is proposed for this rationale is the "thermal endurance profile" (TEP) that is used to drive an endurance coefficient $(B)$ where the higher the endurance coefficient is the greater should be the thermal endurance. One of the basic diagnostic tests to derive these endurance parameters is the aging test that will be carried out on cable specimens to age their plastics without waiting for their failure. According to Dakin [29] the use of so called Arrhenius thermal life equation the 


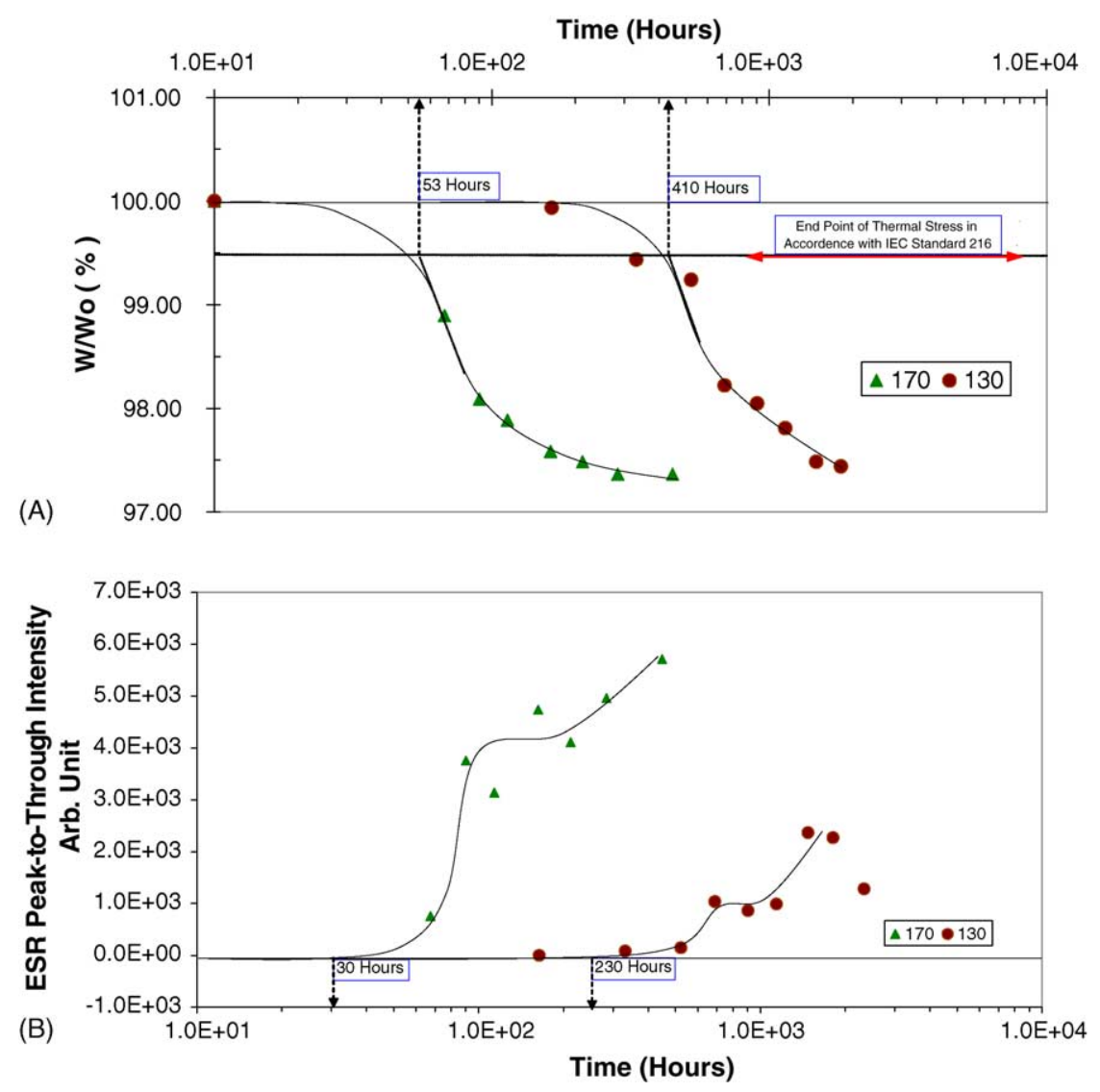

Fig. 3. The onset of the degradation based on weight loss (A) and ESR (B) results at $170^{\circ} \mathrm{C}(\boldsymbol{\Delta})$ and $130{ }^{\circ} \mathrm{C}(\bullet)$ of manufactured red-PVC sample from medium voltage cable. (For interpretation of the references to color in this figure legend, the reader is referred to the web version of the article.)

failure time can be explained using:

$L_{0}=L^{\prime} \exp \left(\frac{B}{T_{0}}\right)$

$B=W / k$, where $W$ is the activation energy, $k$ Boltzman's constant and the higher the activation energy is the greater should be the thermal endurance. $T_{0}$ is the absolute room temperature and $L^{\prime}$ is a constant for the material under consideration that equals the life of material when temperature tends to infinity. Eq. (1) has been modified to represent the "conventional" thermal stress and then can be used for life time analysis $(L)$ at any applied temperature $(T)$ to produce deterioration:

$L=L_{0} \exp (-B c T)$

where $c T=\frac{1}{T_{0}}-\frac{1}{T}=\frac{T-T_{0}}{T T_{0}}$. The conventionality is derived from choosing the room temperature as the reference one, where the thermal stress is explicitly represented by $c T$, which is equal zero at room temperature.

To justify the reliability of the ESR-procedure, thermal aging and hence life curve derivation are investigated for the PVC and XPLE insulation materials using ESR-spectral results and weight loss (IEC Standard method) results. Figs. 3 and 4 show the output of these methods of the manufactured PVC and XLPE, respectively, against aging time.
The time scale in the graph was placed into logarithmic scale because there is a possibility of putting very short and very long times in the same graph and as shown in Eq. (1) the life parameter of thermal stress is of exponential type. Following the IEC Standard 216 of failure criteria ( $0.5 \%$ loss of weight), the end point of thermal stress is identified of all the samples and compared to the onset time of the plastic deterioration on the bases of their radical's contents in the ESR-spectra. The listed results in Table 2 indicates that the weight loss experiment overestimates the predicted failure times compared to those values achieved by ESR experimentation, where the estimated aging time of PVC-samples from weight loss is

Table 2

Failure time in hours of PVC and XLPE insulations based on weight loss and ESR testing

\begin{tabular}{lrrrrr}
\hline Test & Samples & & & \\
\cline { 2 - 6 } & RPVN & RPVR & MPVR & RXLN & MXLN \\
\hline Weight loss & 32 & 37 & 53 & 400 & 350 \\
$\quad$ Failure time at $170^{\circ} \mathrm{C}$ & 32 & 410 & 3000 & 3800 \\
Failure time at $130^{\circ} \mathrm{C}$ & 230 & 380 & & & \\
ESR & & & & & \\
$\quad$ Failure time at $170^{\circ} \mathrm{C}$ & 21 & 23 & 30 & 110 & 60 \\
Failure time at $130^{\circ} \mathrm{C}$ & 120 & 200 & 230 & 1200 & 1050 \\
\hline
\end{tabular}




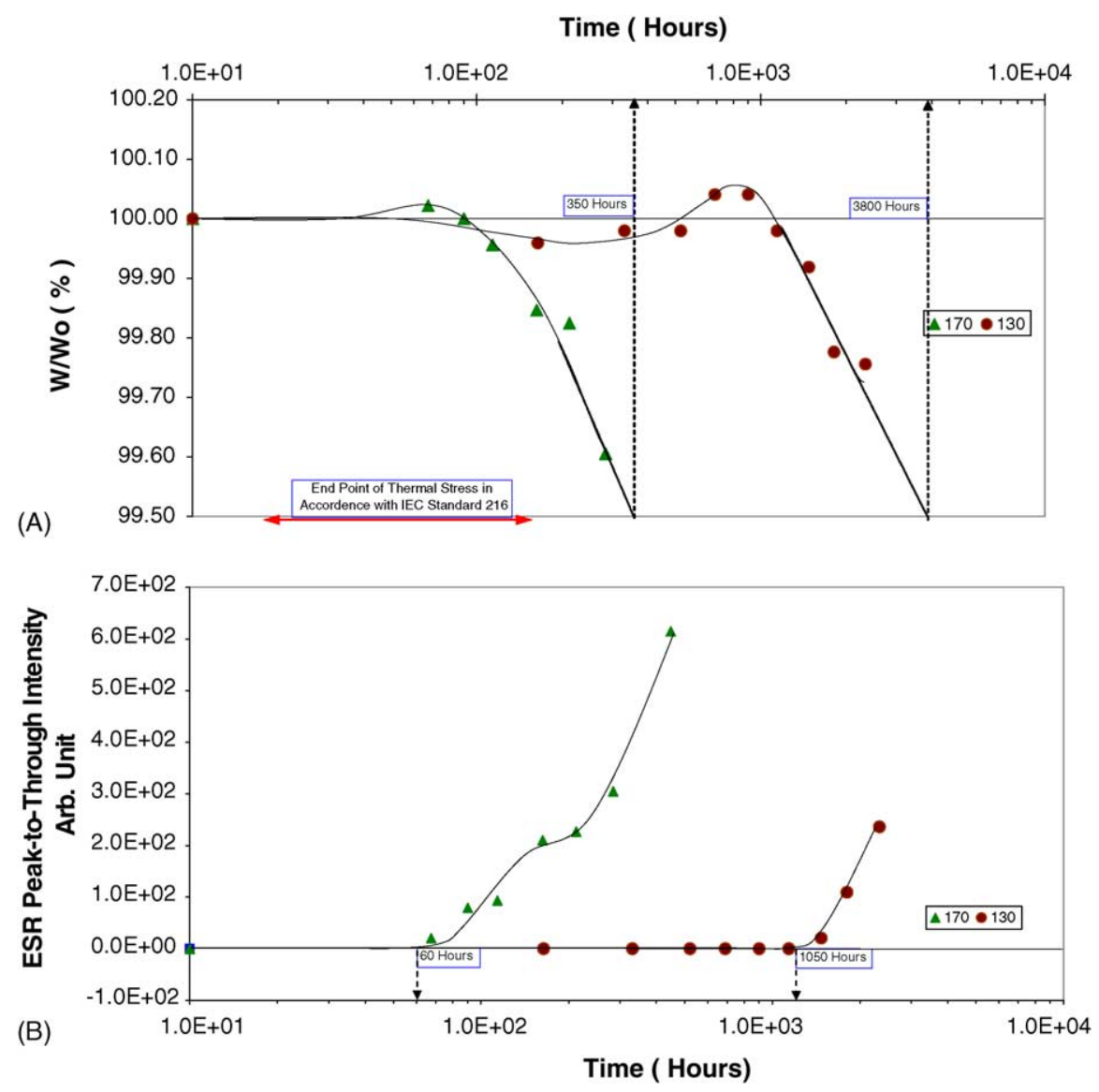

Fig. 4. The onset of the degradation based on weight loss (A) and ESR (B) results at $170^{\circ} \mathrm{C}(\mathbf{\Lambda})$ and $130^{\circ} \mathrm{C}(\bullet)$ of manufactured non-color-XLPE sample from medium voltage cable.

Table 3

Thermal life equation parameters of Arrhenius equation $\left(L_{0}\right.$ and $\left.B\right)$ and temperature index (TI) of PVC and XLPE insulations based on weight loss and ESR tests

\begin{tabular}{|c|c|c|c|c|c|}
\hline \multirow[t]{2}{*}{ Thermal life parameters } & \multicolumn{5}{|l|}{ Samples } \\
\hline & RPVN & RPVR & MPVR & RXLN & MXLN \\
\hline \multicolumn{6}{|l|}{ Weight loss } \\
\hline$L_{0}^{\mathrm{a}}$ & 683578.0 & 4800864.6 & 1641750.9 & 10595559 & 60141090 \\
\hline$B^{\mathrm{a}}$ & 8803.0 & 10396.0 & 9131.2 & 8993.0 & 10644.0 \\
\hline $\mathrm{TI}^{\mathrm{b}}$ & 61.6 & 76.3 & 71.0 & 98.4 & 106.2 \\
\hline $\mathrm{HIC}^{\mathrm{c}}$ & 8.8 & 8.1 & 9.0 & 10.6 & 9.3 \\
\hline \multicolumn{6}{|l|}{ ESR } \\
\hline$L_{0}$ & 140715.3 & 1286773.9 & 888040.3 & 19363066 & 115128243 \\
\hline$B$ & 7726.5 & 9587.7 & 9029.4 & 10593 & 12688.0 \\
\hline TI & 45.6 & 65.0 & 63.4 & 91.3 & 95.7 \\
\hline HIC & 9.0 & 8.2 & 8.6 & 8.6 & 7.4 \\
\hline \multicolumn{6}{|l|}{ Electric strength $^{\mathrm{d}}$} \\
\hline$L_{0}$ & - & - & - & - & 177355330 \\
\hline$B$ & - & - & - & - & 12669.9 \\
\hline TI & - & - & - & - & 101.2 \\
\hline $\mathrm{HIC}$ & - & - & - & - & 7.8 \\
\hline
\end{tabular}

${ }^{\text {a }}$ Using thermal graph slope and intercept.

b The temperature at which the insulation materials will reach the end point, failure criterion, after $20,000 \mathrm{~h}$.

${ }^{\mathrm{c}}$ Halving interval (HIC): the temperature interval, in " $\mathrm{C}$, expressing the halving of the time-to-end-point taken at the temperature corresponding to TI. It is related to life-line slope $(b)$ of Eq. (3): $\mathrm{HIC} \sim(\mathrm{TI}+273)^{2} \log (2 / b)$.

${ }^{\mathrm{d}}$ Ref. [27]. 

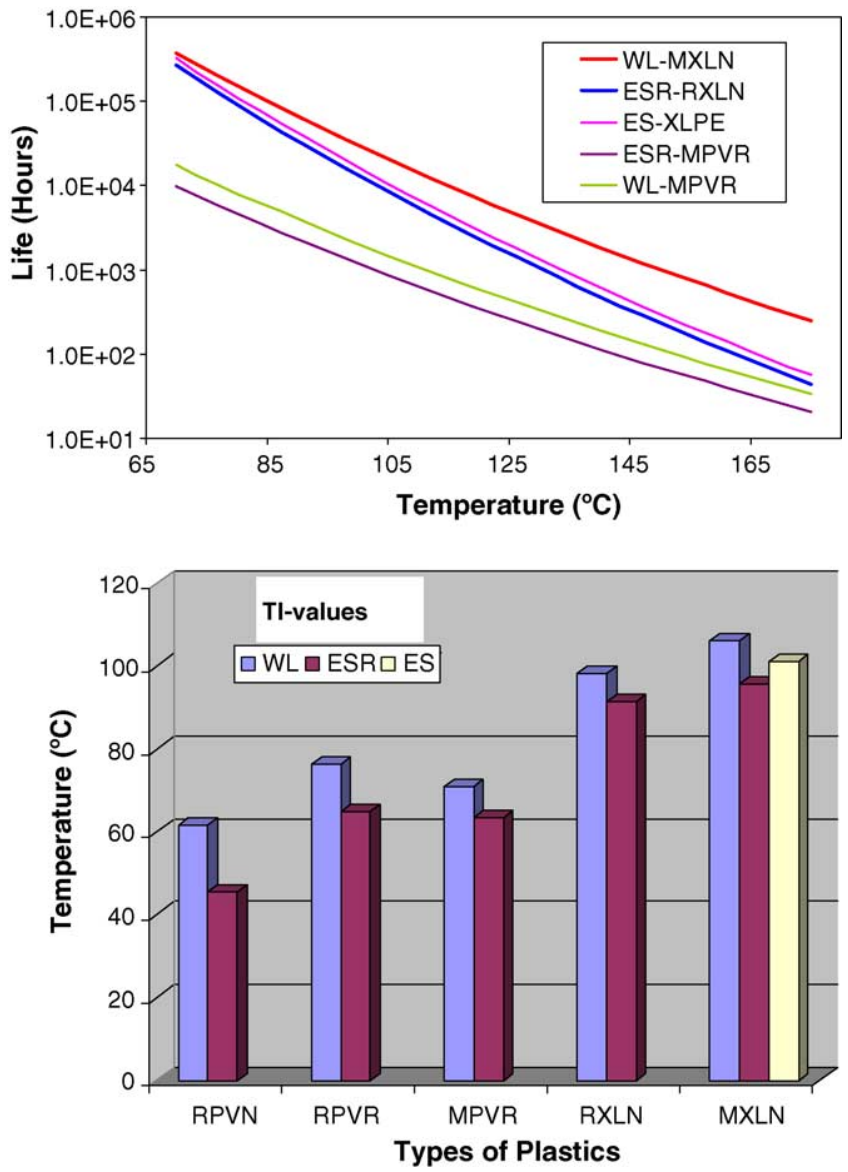

Fig. 5. Life curves (upper graph) and temperature index (lower graph) of manufactured XLPE and PVC systems using different analytical methods: weight loss (WL), ESR and electric strength (ES) tests.

almost twice their correspondence from ESR and it is about three times higher of XLPE-sample at $130^{\circ} \mathrm{C}$ and five times at $170^{\circ} \mathrm{C}$.

Arrhenius graph using the logarithmic form of the "conventional" thermal stress equation:

$\log t_{L}=a+\frac{b}{T}$

has been used to calculate the insulation materials' life parameters: life time at room temperature $\left(L_{0}\right)$, endurance coefficient $(B)$ of the studied systems, the temperature index and halving interval (HIC) values. The obtained values of these parameters are listed in Table 3. In general, the estimated $L_{0}$-values of PVC materials at room temperatures $\left(22^{\circ} \mathrm{C}\right)$ using ESR results is shorter than the one estimated by weight loss, while for XPLE, it is longer. Similar trends are also observed in the values of the temperature endurance coefficient $(B)$. These trends may indicate that two opposite thermal behavior are encountered by the different experimental techniques. However, the life curves of both PVC and XLPE (Fig. 5) clearly indicate that other analytical methods than ESR, e.g. weight loss (WL) or electric strength (ES) [6] overestimates the thermal endurance of the studied samples. This is expected since ESR spectroscopic method, as reported earlier, is the only method able to identify accurately the onset of the polymer deterioration. These results point out that it is not enough to evaluate thermal endurance parameters separately while relationship between temperature and life as illustrated in the "thermal endurance profile" is very important.

\section{Conclusion}

Thermal history of cable insulations is essential criteria to predict cable's life. It consists of two factors: (1) temperature and (2) exposure time. This ESR-spectral result adds third factor molecular properties of the studied samples. Thermal deterioration of the insulation materials over time is a well-known property. The ESR results show clear evidence that the thermal behavior of XLPE insulation is better than PVC insulation that is in good agreement with the well-known properties of XLPE. The results also confirm that colorants enhance the thermal stability of PVC insulating material. Moreover, this analytical procedure is the most direct one yet to pinpoint the starting time of the accelerated thermal degradation. It is also remove the doubt on finding the end-point criterion using different conventional methods, e.g. residual electric strength, tensile strength and/or decrease of weight, to achieve a unique thermal endurance graph, which can be related to degradation and loss of reliability of cables in service conditions. Finally, it has been observed that ESR-measurements can provide thermal endurance characterization consistent with or even better than those obtained by the other conventional methods for any insulation cable models; this could promote a more extensive use of such techniques. In fact, it offers the possibility to investigate the thermal endurance of new materials suitable for cable insulation and based on PVC and XLPE, in relatively short times and accurately. This seems to be an important goal in the light of improving reliability and performance of polymeric insulations for high-voltage electrical applications in cable industry.

\section{Acknowledgments}

This work has been supported by the Research Institute, King Fahd University of Petroleum \& Minerals (KFUPM), under Award of Applied Research Income No. ARI-013 and Jeddah Cables Company who supply the samples. The authors express their appreciation and gratitude to the financial support granted by the KFUPM; also, extend their appreciation to Jeddah Cables Company for their supply of samples and support.

\section{References}

[1] A Technical and Socio-Economic Comparison of Options to Products Derived from the Chlor-alkali Industry, CHEMinfo Services Inc., Canada, 1997. 
[2] T. Fukudu, Z. Iwata, S. Irie, M. Matsuki, S. Kujiki, Y. Takayama, K. Ishihara, Furukawa Rev. 5 (1987) 1.

[3] K. Kobayashi, S. Nakayama, T. Niwa, Proceedings of the Fourth International Conference on Properties and Applications of Dielectric Materials, July 3-8, 1994, p. 678.

[4] T. Kujirai, T. Akagira, Sci. Papers Inst. Phys. Chem. Res. 2 (1925) 223.

[5] G. Mazzanti, G.C. Montanari, L. Simoni, IEEE Electric. Insulat. Mag. 13 (1997) 24.

[6] G.C. Montanari, A. Motori, J. Phys. D: Appl. Phys. 24 (1991) 1172.

[7] D.J. Toop, IEEE Trans. Electric. Insulat. 6 (1971) 2.

[8] B. Yi, Z. Yingsuo, M. Youxiu, Proc. 1st 8 IEEE ICPADM (1985) 218.

[9] Chinese Standard JB1544, 1988.

[10] J.P. Crine, IEEE Trans. Electric. Insulat. 22 (1987) 169

[11] E. Zavoisky, J. Phys. U.S.S.R. 9 (1945) 245.

[12] N.M. Atherton, Principles of Electron Paramagnetic Resonance, Ellis Horwood, London, 1993.

[13] S.N. Batchelor, B. Henningsen, H. Fisher, J. Phys. Chem. 101 (1997) 2969.

[14] W.A. Pryor, B.J. Hales, P.I. Premovic, D.F. Chruch, Science 220 (1983) 425.
[15] S.I. Kuzina, A.P. Pivovarov, G.P. Belov, A.I. Mikhailov, High Energy Chem. 31 (1997) 382.

[16] J.N. Hay, J. Polym. Sci. A18 (1970) 1201.

[17] S.A. Liebman, D.A. Ahlstrom, E.J. Quinn, A.G. Geigley, J.T. Meluskey, J. Polym. Sci. A19 (1971) 1921.

[18] S.A. Liebman, J.F. Reuwer, K.A. Gollatz, C.D. Nauman, J. Polym. Sci. A19 (1971) 1823.

[19] E. Tsuchida, C.N. Shin, L. Shinohara, S. Kambara, J. Polym. Sci. A12 (1964) 3347.

[20] K. Tsuji, Adv. Polym. Sci. 12 (1973) 131.

[21] W.Y. Wen, D.R. Johnson, M. Dole, J. Phys. Chem. 78 (1974) 1798.

[22] E.J. Lawton, J.S. Balwit, R.S. Powell, J. Chem. Phys. 33 (1960) 405.

[23] S. Ohnishi, Y. Ikeda, S. Sugimoto, I. Nitta, J. Polym. Sci. 47 (1960) 503.

[24] S. Ohnishi, S. Sugimoto, I. Nitta, J. Polym. Sci. A11 (1963) 605.

[25] S. Ohnishi, S. Sugimoto, I. Nitta, J. Chem. Phys. 39 (1963) 2647.

[26] A. Motori, F. Sandrolini, G.C. Montanan, IEEE Trans. Power Deliv. 6 (1991) 34.

[27] M. Cacciari, G.C. Montanan, A. Motori, A. Cavallini, L. Simoni, IEEE Trans. Power Deliv. 7 (1992) 634.

[28] IEC Publication 216, third ed., 1989-1990.

[29] T.W. Dakin, Sci. Eng. Ser. 1 (1960) 124. 